STA-MCA Bypass and eight underwent combination of both. Thirteen patients belonged to pediatric age group (age $<18$ years). There were 10 male patients. One patient had an associated cleft lip and palate. Standard monitoring and anesthesia techniques were used. Fourteen patients were induced with intravenous agent while two underwent inhalational induction. Thirteen were maintained on inhalational agents and two on propofol infusion. Opioids and muscle relaxants were used as required. All patients had a hemodynamically stable course. Normocapnia and normothermia was maintained. One patient had significant blood loss requiring transfusion. All patients were reversed and extubated. The mean duration of anesthesia was 2.45 (1:30-4) hours. The mean duration of hospital stay was 4.5 (3-10) days. Two patients had postoperative aphasia. Conclusion: Our data shows that with efficient perioperative anesthetic management the patients with Moyamoya disease can have a favorable outcome. Careful perioperative anesthetic management in Moyamoya disease is associated with good patient outcome.

\section{Comparison of analgesic effect of infusion of low dose ketamine and dexmedetomidine on post-operative pain in spine surgery}

\author{
Nidhi Bidyut Panda, Neha Garg, Y. K. Batra, \\ Hemant Bhagat, R Chabra ${ }^{1}$
}

Department of Anaesthesia and Intensive Care, ${ }^{1}$ Neurosurgery, Post Graduate Institute of Medical Education and Research (PGIMER), Chandigarh, India

Background: The aim of the study was to compare the effects of infusion of Dexmedetomidine and low dose of Ketamine on postoperative pain in patients undergoing SpineSurgery. Materials and Methods: In a randomized, double-blind controlled trial, 66 ASA 1-2 patients undergoing elective lumbar and thoracic spine surgery were divided into three groups. Group K received Ketamine with midazolam bolus $(0.25 \mathrm{mg} / \mathrm{kg}$ and $10 \mu \mathrm{g} / \mathrm{kg})$ followed by infusion of a mixture of Ketamine and Midazolam $(0.25 \mathrm{mg} / \mathrm{kg} / \mathrm{hr}$, $10 \mu \mathrm{g} / \mathrm{kg})$. Group D received dexmedetomidine bolus $(0.5 \mu \mathrm{g} / \mathrm{kg})$ and infusion $(0.3 \mu \mathrm{g} / \mathrm{kg} / \mathrm{hr})$. Group C received normal saline infusion at a rate of 3-8 $\mathrm{ml} / \mathrm{hr}$. All patients received test drug infusion for 24 hours and assessed till 48 hours postoperative period. Morphine $3 \mathrm{mg}$ bolus was used as rescue analgesic and sedation was assessed by Modified Ramsay sedation scale. Results: Pain score differed significantly at 2, 6, 8, 12, 24 and 48 hours postoperatively with the lowest score in group $\mathrm{K}$ then $\mathrm{D}$ and maximum in group $\mathrm{C}$. The pain scores were comparable between group $\mathrm{K}$ and $\mathrm{D}(\mathrm{p}-0.620)$. Rescue analgesic requirement was minimum in group $\mathrm{K}<\mathrm{D}<\mathrm{C}$ (P VALUE-.000). Sedation score was higher in group $\mathrm{K}$. No significant difference in side effects was found in the three groups. Conclusion: Ketamine and dexmedetomidine have comparable analgesic effects. Dexmedetomidine was associated with lower side effects (nausea, vomiting, dizziness, diplopia) than Ketamine, though the difference was not statistically significant. Dexmedetomidine or low dose Ketamine infusion can be used for postoperative analgesia in spine surgery.

\section{Extubation failure in head-injured patients: An analysis of three-months data}

Varun Jain, Sujoy Banik, Ashish Bindra, Keshav Goyal, Girija Prasad Rath

Department of Neuroanaesthesiology, AlIMS, New Delhi, India

Background: Mechanical ventilation is the life sustaining modality, used until the patient is deemed fit for extubation and predicting extubation failure or re-intubation is one of the most challenging. The literature on predictors of successful extubation in head injured patients is very scarce. Hence, we planned to determine the weaning parameters which may predict re-intubation in these patients. Materials and Methods: In this observational study, we present three (3) months data of patients admitted to our neurocritical care unit (NCCU), who were extubated after achieving the standard criteria for tracheal extubation. Data on improvement of Glasgow Coma Scale (GCS) score, duration of mechanical ventilation, $\mathrm{PaO}_{2} / \mathrm{FiO}_{2}$ ratio, hemoglobin, tolerance to spontaneous breathing trials, adequate cough reflex, tracheal secretions and frequency of suctioning were recorded. Pre-extubation criteria such as associated co-morbidities, nature of injury, post-surgical complications (like hematoma, CSF leak, and electrolyte imbalance) were analyzed. Results: Seventy (70) patients who met the inclusion criteria were analyzed; of which $9(12.8 \%)$ patients required re-intubation. Postoperative hydrocephalus, infarction, and impaired gag reflex were found to be the risk factors for re-intubation in these cases $(P<0.05)$. Most of these patients required percutaneous tracheostomy except two with ventilatory failure who were successfully extubated at the second attempt. Conclusion: Our preliminary study suggested that post-procedural neurosurgical complications and impaired gag reflex were the risk factors for re-intubation in head-injured patients. However, a larger study with big sample size may be needed to substantiate these findings.

\section{Effect of hypertonic saline and mannitol on patients undergoing supratentorial tumor surgery}

Kiran Jangra, V. K. Grover, Ankush Singla,

Preethy J. Mathew, S. K. Gupta ${ }^{1}$ 
Department on Anaesthesia and Intensive Care and ${ }^{1}$ Neurosurgery, Postgraduate Institute of Medical Education and Research, Chandigarh, India

Background: To compare the efficacy of equimolar, equivolemic solutions of 3\% hypertonic saline and $20 \%$ mannitol on intraoperative brain relaxation in patients with clinical and radiological evidence of raised ICP undergoing surgery for supratentorial tumors. Materials and Methods: This study was carried out on 30 ASA I-III patients with age group ranging between 18-65 years, undergoing supratentorial tumor surgery. Patients received equimolar, equivolemic solutions of $3 \%$ hypertonic saline (osmolarity-1024) and 20\% mannitol (osmolarity-1098). Both the agents were administered at the dose of $5 \mathrm{ml} / \mathrm{kg}$ over a period of 15 minutes. Patients with previous history of electrolyte imbalance and getting hypertonic saline (HTS) prior to the surgery were excluded from the study. Brain relaxation was assessed by anesthesiologist (on a 3 point scale) and surgeon (on a 4 point scale). Results: Equimolar solutions of both mannitol and 3\% saline produced similar relaxation as assessed by surgeon and anesthesiologist. Urine output was more in mannitol group where as hypertonic saline group had increased serum sodium concentration, which returned to normal in 48 hours. CVP and mean arterial pressure were maintained close to the baseline in HTS group but CVP was higher in mannitol group but returned to normal in 3-4 hours. Conclusions: Since both these agents' mannitol and HTS have nearly equimolar concentration, they produce similar brain relaxation. Gemma et a., $l$ (1997) and Rozet et al., (2007) also had similar observations. Equimolar concentration of HTS and mannitol produce similar brain relaxation in patients undergoing surgery for supratentorial tumors.

\section{Effect of dexmedetomidine on postoperative recovery in patients undergoing cervical spine surgery}

\author{
Varun Jain, Arvind Chaturvedi, Mihir P Pandia, \\ Parmod K. Bithal \\ Department of Neuroanaesthesiology, AIIMS, New Delhi, \\ India
}

Background: The present study is planned to evaluate the effect of dexmedetomidine as an intraoperative anaesthetic adjuvant and its effect on postoperative extubation and recovery profile in patients undergoing anterior cervical spine surgeries. Primary objective was to assess postoperative recovery profile. Secondary objectives were to assess postoperative pain, time for need of first analgesic and to observe the perioperative hemodynamics. Materials and Methods: Thirty ASA I-II Adult patients (age 18-60 yrs) were randomized in this placebo controlled, double blind study. In the Dexmed Group, Dexmedetomidine was started at $0.2 \mu \mathrm{g} / \mathrm{kg} / \mathrm{hr}$ after a loading dose of $1 \mu \mathrm{g} / \mathrm{kg}$ before induction. Perioperative hemodynamics, intraoperative fentanyl and sevoflurane consumption, and postoperative recovery profile were observed by blinded observer. Postoperative pain and discharge readiness from post anesthesia care unit was evaluated using VRS score and modified Aldrete score, respectively. Results: Seventeen patients in placebo and 18 in dexmedetomidine group were enrolled. Time to emergence, extubation and to achieve modified Alderte score $\geq 9$ was earlier in Dexmed group (mean 7.8 minutes; 9.8 minutes; 4.5 minutes) compared to Placebo group (10.5 minutes; 13.2 minutes; 13.7 minutes) $(P=0.01)$. Pain score at extubation was lower (2.8 vs. 26.2) and time for first ${ }^{t}$ analgesic longer (46.6 minutes vs. 18.7 minutes) in Dexmed group compared to placebo. Hemodynamics was better controlled with Dexmedetomidine. Conclusion: Use of Dexmedetomidine in such low dose for abolishing cough and pressor response has not been described previously in cervical spine surgeries where smooth emergence and extubation is desirable. Intraoperative use of dexmedetomidine at lowest recommended dosage in adults undergoing anterior cervical spine surgery results in a favorable recovery profile with reduced emergence/ extubation time and postoperative pain, without adverse perioperative hemodynamic effects.

\section{To evaluate the effects of dexmedetomidine on intraocular pressure and hemodynamic changes in response to laryngoscopy and tracheal intubation and its influences on anesthetic requirements during intracranial tumor surgery}

Tanuja Trivedi, Shobha Purohit

Department of Anaesthesia, SMS Medical College, Jaipur, Maharashtra, India

Background: Brain relaxation is one of the most important prerequisites for neurosurgeries. The other points of concern are the need of stable hemodynamics with less fluctuation in ICP and speedy recovery from anesthesia. Endotracheal intubation is one of the major stressful stimuli inside an operation theatre that can elicit a marked pressor response. Various drugs have been used to attenuate these reflexes and reduce hemodynamic changes. $\alpha_{2}$-Agonist are a novel class of drugs They have neuroprotective, cardioprotective, and sedative effects. These unique characteristics make them potentially useful during neuroanesthesia. Recent studies have shown that Dexmedetomidine is able to decrease circulating plasma norepinephrine and epinephrine concentration 